\title{
Synchronous Colorectal Liver Metastases: Triumph Of Prospective Randomized Trials Over Observational Bias Leads To Paradigm Shift
}

\author{
Vijay P. Khatri, MBChB, FACS \\ University of California School of Medicine, Sacramento, CA
}

"We can't solve problems by using the same kind of thinking we used when we created them."

Albert Einstein

The last one and a half decade has witnessed an exciting era for the management of advanced colorectal cancer, with emergence of effective combination chemotherapy, potent biologic agents, surgical techniques for safe hepatic resection and ancillary procedures such as portal vein embolization and radiofrequency ablation. Integration of these various interventions within the context of a multidisciplinary approach has fostered an aggressive stance amongst oncologists. ${ }^{1}$ This approach continues to be pushed forward with attempts to "tease out" additional patients who may become candidate for curative hepatic resection and hence alter the natural history of their disease. ${ }^{2}$

In this issue, Reddy et al comprehensively review the current evidence for outcomes in the management of synchronous colorectal liver metastases (CRLM) and inquire whether a paradigm shift is necessary. ${ }^{3}$ Let us examine some of the controversial issues that the authors raise in this review and then determine whether a paradigm change is justified.

The traditional approach to the management of the asymptomatic primary tumor with synchronous CRLM has been to resect it to avoid future obstruction, perforation or bleeding. This notion is particularly evident for rectal primaries where surgeons believe that at minimum a diverting colostomy is necessary to prevent eventual obstruction during systemic chemotherapy/chemoradiation. Given that even primary tumors are now commonly considered quite responsive to current multiagent

(C) The Author(s) 2009. This article is published with open access at Springerlink.com

Published Online: 17 March 2009

V. P. Khatri, MBChB, FACS

e-mail: vijay.khatri@ucdmc.ucdavis.edu chemotherapy, potential complications are an unlikely event as evidenced by the report from the BRiTE registry. ${ }^{4}$ Additional evidence will hopefully become available from the National Surgical Adjuvant Breast and Bowel Project (NSABP) C-10, a phase II trial evaluating FOLFOX6 with bevacizumab in patients who present with untreated primary colon cancer and concomitant metastatic disease not considered surgically resectable for cure.

Downsizing to resectable disease has been extensively investigated by the Paul Brousse group in France. Adam et al reported their experience of a multimodality approach where patients received multiple cycles of chemotherapy and, if deemed resectable, then integrating a combination of hepatic resection, resection of extrahepatic disease, portal vein embolization, radiofrequency ablation, cryotherapy, two-stage hepatectomy, and repeat hepatectomy to achieve cure. ${ }^{2}$ When one critically examines this most aggressive approach for CRLM with an intention-to-treat analysis, the percentage of long-term survivors at a mean follow-up of 48.7 months was very low (3.5\% alive and $2.2 \%$ alive without disease). This appears to be a long run for a short slide. When the same data are used to claim that chemotherapy will nullify the biologic effects of portal lymph node metastases, the reservations expressed by Wagman have to be echoed. ${ }^{5}$ Inoperable colorectal metastases may be considered a declining entity, but when one examines the published series on "conversion chemotherapy", patient selection is an important variable and it is clear that, the criteria for non-resectability not only differed between the studies but were often poorly defined.

Until the report of the multi-institutional phase III trial by Portier et al, there had been no clear evidence from a randomized trial that adjuvant chemotherapy, either systemic or by hepatic artery infusion, added benefit over surgery alone. This trial was marred by slow accrual and inadequate sample size (173 of the planned 200 patients over a period of 10 years) but nonetheless did show 
improvement in disease-free survival with the now obsolete 5-fluorouracil (5_FU) and leucovorin. ${ }^{6}$ It thus offered proof of concept for adjuvant chemotherapy in this patient population. This trial was included in a pooled analysis by Mitry et al that showed at best a marginal statistical significance (median progression free survival and overall survival) in favor of adjuvant chemotherapy with a 5-FU bolus-based regimen after complete resection of CRLM. The NSABP trial C-09, which randomly assigned patients to either systemic capecitabine and oxaliplatin alone or with alternating hepatic artery infusion of floxuridine after resection of liver-only metastases, was designed to answer an important question but the study was unfortunately closed as a result of lack of patient accrual-what now appears to be a common theme!

The value of perioperative chemotherapy was evaluated by the phase III prospective randomized controlled trial (PRCT) by Nordlinger et al. ${ }^{7}$ In this study, 364 patients with up to four resectable liver metastases were randomly assigned to either six cycles of fluorouracil, leucovorin, and oxaliplatin (FOLFOX-4) before and six cycles after surgery or to surgery alone. The results demonstrated the absolute increase in rate of progression-free survival with perioperative chemotherapy at 3 years to be $7.3 \%(P=0.058)$ in randomly assigned patients, $8.1 \%$ in eligible patients $(P=0.041)$, and $9.2 \%$ in patients undergoing resection $(P=0.025)$. This benefit was countered by a significantly increased postoperative complication rate in those patients who received perioperative chemotherapy versus surgery alone. These complications included serious ones such as biliary fistula, hepatic failure, intra-abdominal infection, and the need for re-operation. These active chemotherapeutic agents when administered preoperatively have indeed influenced the morbidity of hepatic resection, raising the important question, which needs to be answered in a prospective trial: is adjuvant or neoadjuvant chemotherapy less toxic to the liver? Indeed, an Intergroup US multicenter prospective randomized phase III trial of perioperative chemotherapy versus adjuvant chemotherapy in resectable hepatic metastases is in the planning stage and should be supported by the oncologists.

Most observational studies comparing outcomes of synchronous CRLM to metachronous disease show that survival is not dismal enough to justify a nihilistic approach but we have to remember that the supporting evidence is still Level III. Similarly, the debate about simultaneous colorectal and hepatic resection for colorectal live metastases is marred by the distinct absence of randomized control trials: the evidence Level is II to III with grade $\mathrm{C}$ recommendations. ${ }^{8}$ Almost all authors suggest that a synchronous approach is feasible with the caveat that careful selection of patient ought to be made by surgeons specialized in colorectal and hepatobiliary surgery functioning within a large-volume hospital. Here the adage of Cady is relevant, "Tumor biology is king; and patient selection is queen and technical details of surgical procedures are the princes and princesses of the realm who frequently try to overthrow the powerful forces of the king and queen, usually to no avail, although with some temporary apparent victories". ${ }^{9}$

Having examined the issues raised by the authors, what we have here is a large body of observational studies sprinkled with some prospective randomized trials that forms the basis for suggesting a paradigm shift. Paradigm is a trendy and an overused term, although current usage of this term often has very little to do with what Kuhn put forward. Paradigm shift occurs when Albert Einstein enlightens us with the theory of special relativity, challenging the very concept of time and space to hold that simple Newtonian mechanics are the same for all observers in uniform motion. The same occurred when Bernard Fisher so provocatively challenged Halstedian principles by proposing that breast cancer can be considered a "systemic disease at its inception".

When it comes to management of synchronous CRLM, should we recommend a change in paradigm when there is an overwhelming lack of Level I evidence? The answer is no!- what we need is an ounce of judgment balanced with a pound of commitment to prospective randomized trials. Acceptance of exactly such "paradigm shifts" by the oncology community, based upon low-level evidence, fosters personal biases and results in poor accrual to PRCT that are asking important questions. A concerted effort on designing multi-institutional (adequately and realistically powered) prospective randomized phase III clinical trials and then completing them in a timely fashion with international cross-Atlantic cooperation is necessary to counteract the problems of poor accrual. We do not need yet another retrospective series. Whether it is a single institutional report with 100 patients or a multi-institutional retrospective review with 1000 patients, it does not change the presence of bias nor the fact that the evidence it provides still remains Level III or at best Level II.

So, the real question is, how far do we extend the indication for hepatic resection? The analogy that comes to mind is the Frank-Starling Curve: an increase in preload will increase the cardiac output until very high end diastolic volumes are reached. At this point cardiac output will not increase with any further increase in preload, and may even decrease after a certain preload is reached. Similarly, if we continue to expand the indications, there comes a point where further extension only leads to inferior outcome. Admittedly, surgery is still the one modality that offers the potential of cure, but is likely reaching its limit of benefit. Further enhancement of patient outcomes for this patient population remains a challenge. Whether this might 
be achieved by better harnessing tumor biology, by precise profiling of gene expression, by better disrupting dangerous gene products, or by other methods, remain tantalizing questions in oncology.

OPEN ACCESS This article is distributed under the terms of the Creative Commons Attribution Noncommercial License which permits any noncommercial use, distribution, and reproduction in any medium, provided the original author(s) and source are credited.

\section{REFERENCES}

1. Khatri VP, Petrelli NJ, Belghiti J. Extending the frontiers of surgical therapy for hepatic colorectal metastases: is there a limit? J Clin Oncol. 2005;23:8490-9.

2. Adam R, Delvart V, Pascal G, et al. Rescue surgery for unresectable colorectal liver metastases downstaged by chemotherapy: a model to predict long-term survival. Ann Surg. 2004;240:644-57.

3. Reddy SK, Barbas AS, et al. Synchronous colorectal liver metastases: is it time to reconsider traditional paradigm of management? Ann Surg Oncol. 2009. doi:10.1245/s10434-009-0372-1.
4. Grothey A, Sugrue MM, Purdie DM, et al. Bevacizumab beyond first progression is associated with prolonged overall survival in metastatic colorectal cancer: results from a large observational cohort study (BRiTE). J Clin Oncol. 2008;26:5326-34.

5. Wagman LD. Expanded criteria for surgery for liver metastases: thoughtful science or diamond mining? J Clin Oncol. 2008;26: 3663-4.

6. Portier G, Elias D, Bouche O, et al. Multicenter randomized trial of adjuvant fluorouracil and folinic acid compared with surgery alone after resection of colorectal liver metastases: FFCD ACHBTH AURC 9002 trial. J Clin Oncol. 2006;24:4976-82.

7. Nordlinger B, Sorbye H, Glimelius B, et al. Perioperative chemotherapy with FOLFOX4 and surgery versus surgery alone for resectable liver metastases from colorectal cancer (EORTC Intergroup trial 40983): a randomised controlled trial. Lancet. 2008;371:1007-16.

8. Hillingso JG, Wille-Jorgensen P. Staged or simultaneous resection of synchronous liver metastases from colorectal cancer-a systematic review. Colorectal Dis. 2009;11:3-10.

9. Cady B. Basic principles in surgical oncology. Arch Surg. 1997;132:338-46. 